\title{
Research on Longitudinal Driving Stability of Wheel Hub Motor Driving Electric Vehicle
}

\author{
Yinqiao Gao, Xiong Shu \\ Guangdong University of science and technology, Dongguan 523083, China
}

Keywords: Wheel hub motor; Electric vehicle; Driving; Longitudinal driving; Model; Stability

\begin{abstract}
The wheel hub motor technology is a kind of important technology in automobile, and it is also called as in-wheel motor technology. Its feature is to fully integrate the common power, driving force, and braking device into automobile wheels, which simplifies the mechanical part of electric vehicle. This paper carries out a discussion from perspective of wheel hub motor driving electric vehicle and its stability control, and takes dynamic characteristics of this technology as theoretical basis to carry out a research on longitudinal driving stability of wheel hub motor driving electric vehicle.
\end{abstract}

\section{Introduction}

Compared to the driving force of traditional internal combustion engine, the wheel hub motor has better driving force on electric vehicle, for the wheel hub motor has easier structure and more flexible operation; as for current energy-conservation modern electric vehicle, the wheel hub motor driving solves two social public issues, that is, environmental protection and energy saving, thus the wheel hub motor technology is a core technology for future development of electric vehicle. In 1990s, the Japanese automobile manufacturer Toyota integrated wheel hub technology into modern driving vehicle models, which truly expanded the scope of application of wheel hub technology in modern electric vehicle. In 2011, the Riich X1-EV extended-range electric vehicle which was shown in Shanghai International Automobile Industry Exhibition was the electric vehicle model under China's independent research and development for which the wheel hub motor technology was adopted.

\section{Stability control of wheel hub motor}

\section{Relevant research technology}

Except for environmental protection and energy conservation, the wheel hub motor technology also provides great technological support for automobile performance; for example, the torque of electric vehicle can be independently controlled. This kind of technology is a technology in which the wheel hub is used as the core of wheel; it reasonably assigns the torque of each wheel hub motor, has dynamic control which is more complicated than traditional automobile but can be easily realized, and its final purpose is to improve the stability of wheel hub motor driving electric vehicle. The torque of wheel hub motor is characterized by rapid response; therefore, while the wheels are under the state of high-speed slipping, the torque of wheel hub motor can easily control the wheel, realize the highest-performance driving skid-resisting braking, and ensure that the automobile can be rapidly and accurately fixed; in this way, this technology helps to form excellent adhesive ability between wheels and pavement and realize the high driving stability of electric vehicle.

In Japan, the professor Hori Yoichi of University of Tokyo has carried out large quantity of theoretical research and practical operation on driving stability of wheel hub motor driving vehicle. He thinks that it is able to realize the direct yaw moment control of wheel hub motor on automobile by use of the yaw moment caused by the difference in power-driven wheel torque, which is also the professor Hori Yoichi's core theory of wheel hub motor driving dynamic control. In terms of driving skid-resisting control, he has designed the anti-electromotive force observer which depends on wheel hub motor to obtain the wheel rotation and wheel angular acceleration while the wheels slip. Another 
professor Hiroshi Fujimoto has studied the stability control moment of wheel hub motor driving electric vehicle movement based on yaw moment observer. He has found that the driving control of automobile wheels have two aspects, that is, inner-loop control and outer-loop control; the inner-loop control controls the driving force of motor automobile wheels, and the outer-loop control controls the stability of yawing movement of wheel hub motor. Due to the fact that two kinds of control can cause the robustness change on pavement state, he also has studied the yawing dynamic equation based on lateral and longitudinal direction, and has made use of least square solution method to classify lateral and longitudinal force of each wheel so as to realize reasonable balance of lateral and longitudinal force and finally improve the operating stability of vehicle upon steering driving.

The professors of Jilin University also have made contribution on the coordination control of torque of wheel hub motor driving electric vehicle. They think that the principle of wheel hub motor driving electric car is to utilize driving speed and front wheel angle feedforward control to carry out full control on movement state of car body based on centroid offset error feedback of yaw-angle wheel rotation, and obtain the torque feedback coefficient of automobile under the optimal state; make use of the rate of change of adhesion coefficient between wheel and pavement and the change of slip rate of car body to detect the slipping state of wheels, and then obtain the maximum pavement adhesion coefficient slip rate, and finally calculate the stability of vehicle driving by use of slip rate.

\section{Features of wheel hub motor technology}

Through above experts' research on the torque of wheel hub motor driving vehicle, we can summarize out several features of wheel hub motor technology.

Firstly, the direct driving of wheel hub motor on the torque of automobile wheel can let the automobile save the function of clutch and transmission, and improve the transmission efficiency of automobile power to maximum degree. While the transmission system is simplified, the whole car body can realize light weight.

Secondly, the wheel hub driving electric vehicle can depend on the motor to complete the driving control independently, which greatly reduces the content of mechanical accessories in the wheels; this technology has gradually replaced the dynamic control method through which the system is controlled via driving the tractive force.

Finally, the wheel hub motor driving realizes the electronic automation of automobile chassis system. The automobile loaded with wheel hub motor driving can recognize and control the driving force of each wheel through actual driving condition so as to realize easier driving and greatly improve the driving safety.

\section{Relationship between movement state of vehicle and stability}

While the vehicle steers, the centrifugal force will happen due to curvilinear motion, and such centrifugal force will act on each wheel. At this time, the lateral deflection will appear in tire of wheel due to flexibility. With the increase of radius of turning circle, there will be increase in the proportion between lateral force and lateral deflection. Therefore, at the beginning of automobile design, we will consider the lateral deflection rigidity of front and rear wheels of vehicles. The rear axle of automobile shall have a larger lateral deflection rigidity than the front axle so that the deflection of front axle can be larger than that of rear axle upon steering, that is, the understeering feature. Generally speaking, while a car has understeering feature, it means that it has high driving stability, for it can easily modify its steering moment and ensure to avoid various kinds of instability under the condition of complicated operation.

For example, while the automobile steers, the longitudinal and lateral acceleration of automobile body will increase; at this time, the load axle of wheel shall be re-assigned; in this assignment process, the automobile may be under imbalanced stated due to excessive steering; for another example, while the yaw velocity of vehicle is less than the turning speed of front wheel, the yaw moment will cause the increase of side slip angle due to small angle of rotation of front wheel; the larger the side slip angle is, the easier for the vehicle loses the stability. 


\section{Control strategies for longitudinal driving stability of wheel hub motor driving electric vehicle}

The wheel hub motor driving can greatly help to control the longitudinal driving stability of electric vehicle; after a brief research on the relationship between stability control of wheel hub motor driving and vehicle state, we shall combine with different control means to research the strategies for vehicle longitudinal driving anti-slip control of wheel hub motor driving.

\section{Demand of longitudinal force}

Prior to the research on longitudinal driving anti-slip control, we shall firstly know the demand of wheel hub motor driving electric vehicle for longitudinal force. In general, the demand of longitudinal force fully depends on target speed and longitudinal acceleration. This paper applies PID, that is, proportion integration differentiation, to realize the control on longitudinal force.

According to PID principle, we can summarize the control rule of longitudinal force as below:

$$
U(t)=K p\left[e(t)+\frac{1}{T i} \int_{0}^{t} e(t) d t+T d \frac{d e(t)}{d t}\right]
$$

In the above formula, Kp refers to proportionality coefficient, Ki refers to integral coefficient, and Kd refers to differential coefficient; Ti and Td refer to specific time dimension, and they are called as integral time and differential time respectively.

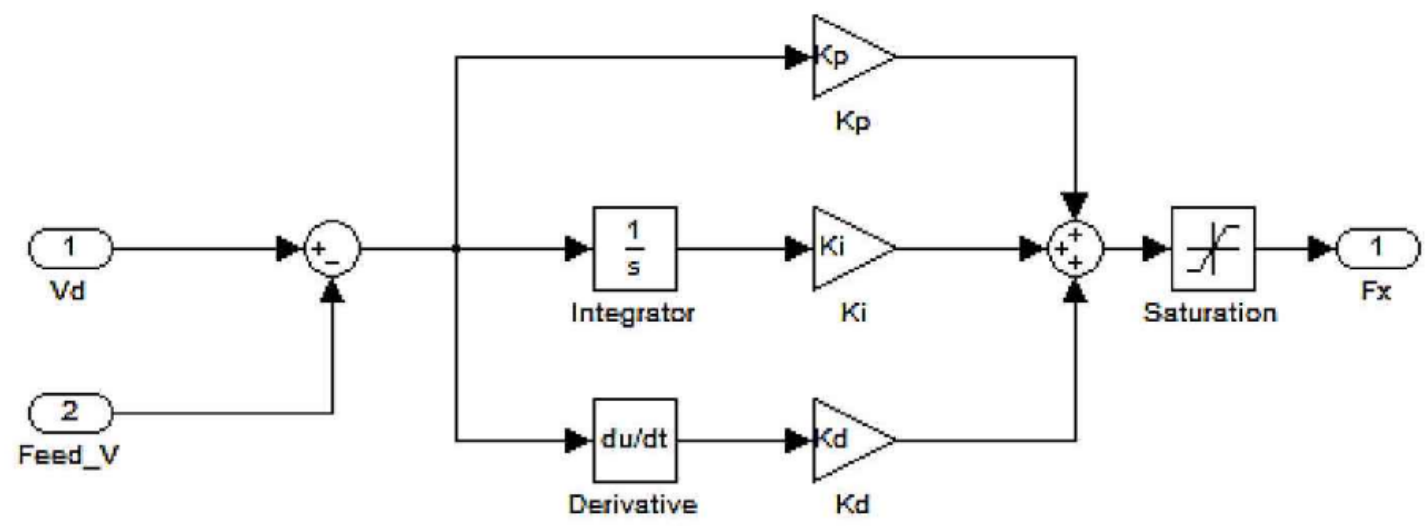

Fig 1. Simulation diagram of vehicle speed control module

As shown in Figure 1, while we use PID to carry out control and regulation on target speed and longitudinal acceleration, the response time of longitudinal driving anti-slip control will be shortened; at this time, it is able to meet the basic demand of wheel hub motor driving electric vehicle on longitudinal force, and then judge the driving stability of longitudinal torque.

\section{Strategies for longitudinal driving stability control of electric vehicle}

The wheel hub motor driving electric vehicle abandons the drive system in traditional vehicles, that is, clutch, transmission, main reducing gear, and differential mechanism. Through the motor loaded in the wheel, it is able to directly form driving force to drive the vehicle. The driving force control system of wheel hub motor consists of two parts of control structure, that is, single-wheel driving anti-slip layer and driving torque distribution layer. Under the control influence of stable driving system, the wheels can stably adhere to the ground, thus the driving safety can be ensured. When the vehicle drives under poor pavement condition, it can also smoothly start and move forward. However, at the starting stage of vehicle, the non-slip wheel driving force will can't be fully exerted due to insufficient starting speed, thus partial vehicle power will lose. Meanwhile, in order to overcome poor pavement condition, the single-wheel driving anti-slip control is started, which causes the situation that the additional yaw moment appears in vehicle and the longitudinal driving stability of vehicle will be influenced. Therefore, we shall consider to utilize wheel hub motor driving electric vehicle single-wheel torque method to improve the vehicle stability under condition of driving anti-slip control. Based on the stability of vehicle longitudinal driving, this paper designs multi-wheel driving torque coordinated control strategy, and constructs vehicle driving anti-slip system and torque coordinated system based on yaw velocity and slip rate which influence the vehicle stability. 
Firstly, the wheel longitudinal force shall be calculated, and the calculation formula is Fxmax $=\mu \max * \mathrm{FZi}$.

According to the formula, we can see that the longitudinal force is restricted by ground adhesion condition. If the torque coordinated system of vehicle controls the yaw moment by use of regulating torque increment, the output of driving torque shall be calculated based on the requirement of ground adhesion under control condition of driving anti-slip system. While the wheel driving reaches the maximum limit of ground adhesion, the longitudinal force shall be controlled via reducing yaw moment at opposite direction. For example, if there is insufficient steering upon vehicle turning and the outer wheels have reached adhesion limit, we shall ensure the stability of vehicle via adopting the control method of reducing rear wheel driving torque. Therefore, while the vehicle is under four-wheel driving, the driving control system must ensure that the torque coordinated control shall be linked with driving anti-slip control system so as to meet the safe and stable requirement of vehicle driving to the maximum degree.

Then, the yaw moment can be directly changed via changing the driving force of vehicle wheels, thus the torque coordinated control has two tasks in the operation process. The first task is to carry out coordinated control on vehicles which take the traditional gas system as driving force to ensure that the vehicles are under both stability control and dynamic control, thus the wheel hub motor driving can be widely applied in modern electric vehicle; the second task is to ensure that each tire of vehicle has the anti-slip control ability, which is the most intuitive protection on vehicle stability. In this link, the wheel hub motor can realize the advantage of rapid control of torque, and improve power performance and flexibility while the vehicle stability increases.

\section{Coordinated control on vehicle torque}

The torque coordinated control plays an important role in the longitudinal driving stability control of vehicle, which shows that the wheel hub motor shall actively improve the acceleration perform upon driving electric vehicle. This condition is especially prominent on some pavements with bad environment. While the vehicles drive on snowy road, the excessive track slip or idle rotation often happens in wheels, which causes the situation that the tractive force of driving wheels continuously decreases and finally the vehicle even flames out due to reduction of vehicle acceleration. Therefore, if we install anti-slip controller in the wheels, it is able to avoid the occurrence of above situation and ensure that each wheel can provide the maximum tractive force instead of causing slipping. At this time, if we use torque coordinated control and control the driving force of four driving wheels at minimum value, we can ensure the coordinated control of vehicle torque on each driving wheel, and then ensure the driving stability.

While the vehicles are under different driving states, there will be different torque coordinated control schemes, according to which we can determine the key point of vehicle performance requirement under different driving states. From Figure 2, we can see that the wheel hub motor driving will change the driving anti-slip steps due to different driving conditions; meanwhile, the torque distribution is also affected by the change in the driving states of vehicles.

While the vehicle is under the control process of starting speed, the vehicle wheels have a low-speed driving with high adhesion to road. At this time, the main purpose of driving the anti-slip control is to make use of the adhesive force of driving wheel to ground to obtain maximum tractive force. On the contrary, if the vehicle drives on low-adhesion road such as ice-snow road, it is required to reduce the driving torque of four driving wheels of the vehicle and quickly adopt braking measures to ensure no slipping of wheels, and make full use of weak adhesive force to ground to gradually improve the acceleration performance of vehicle under stable state and ensure the absolute stability of vehicle. 


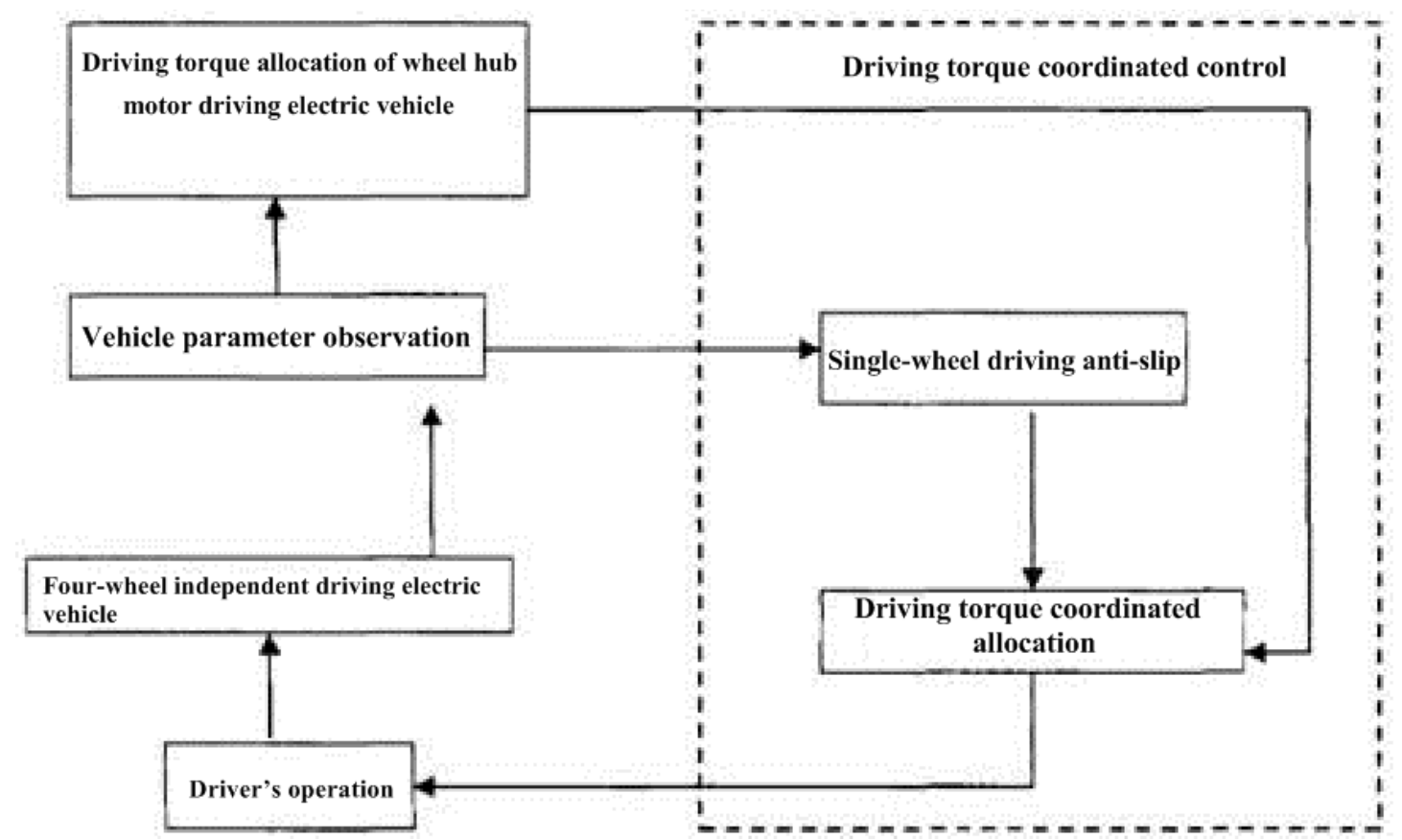

Fig 2. Flow chart for wheel hub motor driving vehicle and torque coordination module control

If the vehicle is under high-speed driving state, the only factor which shall be considered is stability of driving direction of vehicle. At this time, the purpose of driving anti-slip control is just to ensure that four driving wheels can produce same tractive force. The specific practice is to stabilize the slip rate of driving wheel, and ensure that the horizontal adhesive coefficient of wheels can be up to maximum value so that the vehicle can have sufficient ability against sideslip. Therefore, while the vehicle is under high-speed driving state, it is better to adopt multi-driving wheel mode to ensure that the front outer wheels and rear inner wheels with the highest operation efficiency at this time are under the scope of anti-slip control, and carry out auxiliary control on other two driving wheels to realize the absolute control on the longitudinal stability of vehicle, as shown in Figure 3.
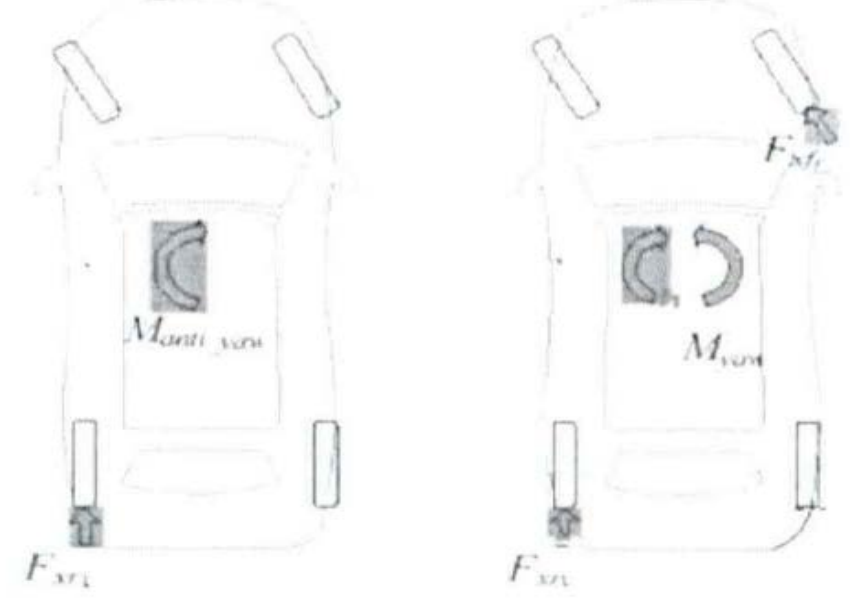

Fig 3. Diagram for regulation of driving force of wheel hub motor driving multi-wheel control mode

\section{Conclusion}

In conclusion, the wheel hub motor driving takes the control on longitudinal stability of vehicle as basic objective, and the final purpose of adopting anti-slip control, torque coordinated control, or other different control modes is to ensure vehicle safety, and especially ensure the drivers' life safety. This paper just analyzes the driving technology of wheel hub motor from one perspective; actually, there are many theoretical practices which involve this field, such as construction of simulation 
platform based on wheel hub motor driving. The further research and exploration are required in future work and study.

\section{References}

[1] Wan Rong: Research on Stability and Fault Tolerance of Electronic Differential of Electric Vehicle, Jiangsu University, 2014.

[2] Sheng Chen: Research on Longitudinal Stability Control of Wheel Hub Motor Driving Electric Vehicle, Beijing Jiaotong University, 2014.

[3] Jiang Zaoyun: Research on Allocation Algorithm of Longitudinal Force of Stability Control of Four-wheel Electric Vehicle, Chinesisch-Deutsches Hochschulkolleg, Tongji University, 2008. 